\title{
DURA LEX SED LEX: OPINIONES SOBRE LA IGUALDAD ANTE LA LEY EN ESPAÑA
}

\author{
DURA LEX SED LEX: OPINIONS ON EQUALITY AND THE LAW \\ IN SPAIN
}

\author{
Paloma Alaminos-Fernández \\ Universidad de Alicante (Spain) \\ paloma.alaminos.fernandez@gmail.com
}

Antonio Alaminos-Fernández

Universidad de Alicante (Spain)

antonio.alaminos@ua.es

\section{Resumen}

Este artículo recoge una investigación sobre las opiniones acerca de la igualdad ante la ley de los españoles. Responde, de forma empírica a las preguntas ¿Considera la sociedad española que el poder judicial actúa con imparcialidad tratando por igual a todos los ciudadanos independientemente de sus características personales? ¿Puede considerarse que existe una discriminación positiva en la aplicación de la justicia, en el sentido de atenuar las consecuencias de la aplicación de la ley en individuos o grupos sociales? ¿Puede considerarse que existe una discriminación negativa en la aplicación de la justicia, en el sentido de agravar su aplicación en los individuos o grupos sociales con características socialmente desfavorables? Y en todo caso, ¿Qué grupos se ven favorecidos o perjudicados por dicha administración desigual de la justicia?

Para ello, en un primer momento consideraremos la opinión sobre la igualdad ante la ley para posteriormente considerar los factores que, según la opinión pública, introduce asimetrías en el tratamiento de la justicia. Finalmente se efectúa un análisis dimensional para agrupar, mediante un análisis factorial, aquellos rasgos o características que según la opinión pública asociación 
discriminación ante la ley. Los datos empleados son los procedentes de dos estudios de opinión pública realizados por el Centro de Investigaciones Sociológicas en septiembre de 2013 y de 2016.

\title{
Palabras clave
}

Opinión pública, igualdad, justicia, discriminación

\begin{abstract}
This article presents an investigation on the opinions on the equality before the law of the Spaniards. The research responds empirically to the questions. Does Spanish society consider that the judiciary acts impartially, treating all citizens equally regardless of their personal characteristics? Can it be considered that there is positive discrimination in the application of justice, in the sense of mitigating the consequences of the application of the law? It can be considered that there is a negative discrimination in the application of justice, in the sense of aggravating its application in individuals or social groups with socially unfavourable characteristics? And in any case, what groups are favoured or harmed by this unequal administration of justice?

For this, at first, we will consider the opinion on equality before the law, to later consider the factors that, according to public opinion, introduce asymmetries in the application of justice. Finally, a dimensional analysis is carried out to group, by means of a factorial analysis, those traits or characteristics that, according to the public opinion, discriminate before the law. The data used are those from two public opinion studies conducted by the Center for Sociological Research in September 2013 and 2016.
\end{abstract}

\section{Key words}

Public opinion, equality, justice, discrimination

\section{Extended abstract}

Equality before the law and the impartial application of justice are two fundamental elements in modern rule of law. In this analysis of Spanish public opinion, it is observed that the majority perception of Spaniards is of a reality where justice is applied unequally. The determining factor is the economic capacity of citizens. It is thought that the rich are favored and the poor are hurt. Together with this factor of inequality before the courts, other characteristics that could influence both favorable and unfavorable terms of justice are analyzed. 
The analysis shows how, from the point of view of public opinion, there are two perceived sources of social discrimination: the differentiation between outgroup and endogroup, and within the endogroup the internal inequalities with respect to a culturally idealized pattern.

The dimensional analysis of the unequal performance of justice according to the characteristics of individuals shows a structure that is close (although methodologically incomparable) to that of society in general. In the area of justice, the presence of the "normality factor" (factor 3) stands out, which identifies men, heterosexuals, Catholics and Spaniards as the least susceptible to being discriminated against. The other groups are defined by belonging to the outgroup from the cultural point of view (factor 2), by their personal characteristics that can be considered not yet fully normalized (factor 1), by poverty (factor 4 ) or by the weight they have within of the social structure to be young or old (factor 5). The woman appears classified almost indistinctly in the factor of individual traits (sex) or in that of weak social position (Age).

In this sense, the perception of an unequal application of the law makes it possible to identify the identifying characteristics of the "normalized" citizen. Thus, the least discrimination of justice would occur for the following attributes: being young, male, Spanish, heterosexual and Catholic, as well as having economic resources. This would be the profile of the group that is considered less discriminated when judges apply the law.

In a society that considers that certain characteristics discriminate against individuals, justice is perceived within that reference system, as non-impartial. In a way, beyond the reality or not of this unequal application of the law, the perception of society defines a situation of delegitimization of the institution from the moment that a percentage as high as $78.2 \%$ in 2016 considers that the law is not the same for everyone.

\section{LA DISCRIMINACIÓN EN CONTEXTO: LA SOCIEDAD}

La discriminación de individuos o grupos sociales se produce en un contexto cultural y social concreto. En ese sentido, tanto la percepción como la intensidad de dichas discriminaciones deben de pasar el filtro de la visibilización. La sociedad debe ser sensible y reconocer como tales dichas situaciones de discriminación. Esta situación diferencial y no igualitaria se extiende por toda la sociedad y alcanza y se basa en rasgos y características cuya normalidad evita que se perciban. Es en dicho contexto cultural y de lo que se considera como socialmente aceptado, que actúan y toman sus decisiones las instituciones. La influencia del entorno en la toma de decisiones es algo que en mayor o menor grado permanece presente. 
Es por este motivo que una de las primeras observaciones debe dirigirse a la percepción que tienen los individuos sobre la presencia de discriminación en función a características concretas. Características que por su "normalidad" pueden permanecer invisibles. Es el caso del sexo, el origen étnico o racial, la nacionalidad, la orientación sexual, la identidad sexual (ser transexual), edad, su religión o creencias religiosas, el aspecto físico, la pobreza (tener pocos recursos económicos), la discapacidad física, la discapacidad psíquica, una enfermedad crónica o infecciosa (hepatitis, diabetes, VIH/Sida) o incluso el tener determinadas ideas políticas. Para ello vamos a considerar la pregunta que efectúa el CIS en el estudio de 2016 analizado dicha realidad social.

P.9 Se dice que una persona (o grupo de personas) es discriminada cuando es tratada de forma más desfavorable que otra debido a sus características personales. En su opinión, en España en general, ¿es muy frecuente, bastante frecuente, poco frecuente o nada frecuente, la discriminación o el trato desfavorable en cualquier ámbito por motivo de...?

Así, en 2016 se observa como la principal característica fuente de discriminación es el origen étnico o racial $(64 \%)$, seguido de tener una discapacidad psíquica $(60 \%)$, tener pocos recursos económicos (56\%), la edad (55\%), el aspecto físico $(55 \%)$, la identidad sexual (ser transexual) (51\%), el género (Sexo) (50\%), tener una discapacidad física (50\%), la nacionalidad (49\%), el tener una enfermedad crónica o infecciosa (hepatitis, diabetes, VIH/Sida) (47\%), la orientación sexual $(42 \%)$, la religión o creencias religiosas (33\%) y finalmente como la menor causa de discriminación el tener determinadas ideas políticas (32\%).

Destacan por lo tanto como más significativos, con un porcentaje superior al $50 \%$ de los españoles que lo opinan, el origen étnico o racial, las discapacidades psíquicas, la pobreza, la edad, el aspecto físico o la identidad sexual. Todas ellas tienen una historia bien conocida detrás. La xenofobia o el racismo, el culto a la belleza y la juventud en las sociedades modernas, el miedo a lo atípico en general. En dicho cúmulo de características cabe destacar la pobreza. La pobreza como causa de discriminación en las sociedades de consumo y del ocio. No cabe en estas páginas desarrollar las causas y contextos históricos que dan forma y explican esas fuentes de discriminación.

Sí importa especialmente, tenerlas presentes como marco de referencia para el estudio de las posibles discriminaciones en ámbitos de los que se espera estricta neutralidad: la justicia. 
Dura lex sed lex: opiniones sobre la igualdad ante la ley en España

Tabla 1. Opinión sobre la frecuencia con la que se discrimina según diversas características de los individuos

\begin{tabular}{|c|c|c|c|c|c|c|c|}
\hline & $\begin{array}{l}\text { Muy } \\
\text { frecuente }\end{array}$ & $\begin{array}{l}\text { Bastante } \\
\text { frecuente }\end{array}$ & $\begin{array}{l}\text { Muy o } \\
\text { bastante }^{1}\end{array}$ & $\begin{array}{l}\text { Poco } \\
\text { frecuente }\end{array}$ & $\begin{array}{l}\text { Nada } \\
\text { frecuente }\end{array}$ & N.S. & N.C. \\
\hline Origen étnico o racial & $15,4 \%$ & $48,6 \%$ & $64,0 \%$ & $23,4 \%$ & $8,4 \%$ & $3,5 \%$ & $0,6 \%$ \\
\hline $\begin{array}{l}\text { Tener una } \\
\text { discapacidad psíquica }\end{array}$ & $15,9 \%$ & $43,9 \%$ & $59,8 \%$ & $22,8 \%$ & $11,9 \%$ & $5,1 \%$ & $0,4 \%$ \\
\hline $\begin{array}{l}\text { Tener pocos recursos } \\
\text { económicos }\end{array}$ & $15,9 \%$ & $40,4 \%$ & $56,3 \%$ & $24,7 \%$ & $15,2 \%$ & $3,4 \%$ & $0,4 \%$ \\
\hline Edad & $13,0 \%$ & $41,9 \%$ & $54,9 \%$ & $27,4 \%$ & $14,8 \%$ & $2,5 \%$ & $0,4 \%$ \\
\hline Aspecto físico & $15,6 \%$ & $39,1 \%$ & $54,7 \%$ & $28,0 \%$ & $14,2 \%$ & $2,5 \%$ & $0,6 \%$ \\
\hline $\begin{array}{l}\text { Identidad sexual (ser } \\
\text { transexual) }\end{array}$ & $13,7 \%$ & $37,0 \%$ & $50,7 \%$ & $25,6 \%$ & $13,6 \%$ & $9,1 \%$ & $0,9 \%$ \\
\hline Sexo & $11,5 \%$ & $38,9 \%$ & $50,4 \%$ & $33,4 \%$ & $13,2 \%$ & $2,4 \%$ & $0,5 \%$ \\
\hline $\begin{array}{l}\text { Tener una } \\
\text { discapacidad física }\end{array}$ & $11,3 \%$ & $39,1 \%$ & $50,4 \%$ & $29,3 \%$ & $16,1 \%$ & $3,8 \%$ & $0,3 \%$ \\
\hline Nacionalidad & $10,8 \%$ & $37,9 \%$ & $48,7 \%$ & $32,1 \%$ & $14,9 \%$ & $3,6 \%$ & $0,7 \%$ \\
\hline $\begin{array}{l}\text { Tener una } \\
\text { enfermedad crónica o } \\
\text { infecciosa (hepatitis, } \\
\text { diabetes, VIH/Sida) }\end{array}$ & $12,2 \%$ & $35,0 \%$ & $47,2 \%$ & $25,9 \%$ & $15,0 \%$ & $11,5 \%$ & $0,5 \%$ \\
\hline Orientación sexual & $9,0 \%$ & $33,3 \%$ & $42,3 \%$ & $33,9 \%$ & $17,1 \%$ & $5,9 \%$ & $0,8 \%$ \\
\hline $\begin{array}{l}\text { Religión o creencias } \\
\text { religiosas }\end{array}$ & $6,4 \%$ & $26,6 \%$ & $33,0 \%$ & $34,0 \%$ & $27,0 \%$ & $5,0 \%$ & $1,0 \%$ \\
\hline $\begin{array}{l}\text { Tener determinadas } \\
\text { ideas políticas }\end{array}$ & $8,2 \%$ & $24,2 \%$ & $32,4 \%$ & $30,6 \%$ & $26,5 \%$ & $9,5 \%$ & $0,9 \%$ \\
\hline
\end{tabular}

Fuente: Elaboración propia con datos del estudio CIS E3150 septiembre de 2016

\section{LA PERCEPCIÓN DE UNA JUSTICIA DESIGUAL}

Los estados de derecho actuales se basan en dos principios axiales que dan forma básica al aparato del estado. Uno de ellos es de carácter organizativo: el principio de división de poderes postulado por Montesquieu. El equilibrio de los poderes del estado se logra en base a contrapesos entre los poderes judicial, ejecutivo y

1 Suma de las categorías mucho y bastante 
legislativo. Tres poderes independientes entre sí para vigilarse y proteger lo que son los valores rectores de la vida ciudadana. El segundo refiere a los valores que debe inspirar la acción del estado en relación con los ciudadanos: la defensa de la libertad, la protección de la igualdad y la garantía de fraternidad social. Con origen en la ilustración se encuentran prácticamente extendidos y reconocidos en los estados democráticos del mundo.

La ley, en los estados sociales y de derecho modernos, se apoya de forma fundamental en la noción de igualdad. La igualdad ante la ley es, lógicamente, un principio definitorio de la noción de justicia.

La Declaración Universal de los Derechos humanos en su artículo 2, afirma "Toda persona tiene todos los derechos y libertades proclamados en esta Declaración, sin distinción alguna de raza, color, sexo, idioma, religión, opinión politica o de cualquier otra índole, origen nacional o social, posición económica, nacimiento o cualquier otra condición". Insistiendo en la igualdad en otros muchos artículos, especialmente el artículo 7 donde "Todos son iguales ante la ley y tienen, sin distinción, derecho a igual protección de la ley. Todos tienen derecho a igual protección contra toda discriminación que infrinja esta Declaración y contra toda provocación a tal discriminación" o el artículo 10 "Toda persona tiene derecho, en condiciones de plena igualdad, a ser oída públicamente y con justicia por un tribunal independiente e imparcial, para la determinación de sus derechos y obligaciones o para el examen de cualquier acusación contra ella en materia penal". La Declaración de Derechos Humanos es fuente de derecho inspiradora de multitud de leyes y tratados internacionales. La igualdad, como puede apreciarse, es una de las garantías a proteger con especial atención. En línea con lo anterior, y destacando para el caso español, la Constitución Española de 1978 en su artículo 14 afirma "Los españoles son iguales ante la ley, sin que pueda prevalecer discriminación alguna por razón de nacimiento, raza, sexo, religión, opinión o cualquier otra condición o circunstancia personal o social". Este principio es reiterado y protegido por multitud de normas y jurisprudencia. En sí mismo, la igualdad ante la ley es un bien superior que debe ser protegido y garantizado.

Si bien desde el punto de vista jurídico del estado moderno la igualdad es un axioma indiscutible, una cosa diferente es la concreción en la experiencia de la sociedad. Una experiencia que se refleja tradicionalmente en la cultura (como son los ejemplos de los refranes) o contemporáneamente en la opinión pública. En ese sentido, frente a la irrefutable afirmación de que todos los españoles somos iguales ante la ley, la percepción y experiencia que refleja la opinión pública es bien diversa. Bastante más próxima a la afirmación de Platón cuando planteaba que "la justicia no es otra cosa que la conveniencia del más fuerte".

El texto que aquí consideramos trata de uno de los poderes, el judicial, y de uno de los valores, la igualdad. La pregunta es única y explícita ¿Considera la 
sociedad española que el poder judicial actúa con imparcialidad tratando por igual a todos los ciudadanos independientemente de sus características personales? Es a esta pregunta a la que damos respuesta en este artículo.

Para ello, continuamos indagando en dos estudios de opinión pública realizados por el Centro de Investigaciones Sociológicas en septiembre de 2013 y de 2016. En primer lugar, consideraremos si la opinión pública piensa que la justicia da un trato igual a los ciudadanos o por el contrario es desigual. La pregunta es la siguiente:

¿Cree que en España a la hora de aplicar las leyes se da el mismo trato a todas las personas, o se hacen diferencias según de quien se trate? Tiene como respuesta dos opciones posibles: se da el mismo trato a todas las personas o se hacen diferencias según de quien se trate.

Así cuándo se pregunta con respecto a si la aplicación de las leyes en España es igual para todos o por el contrario existen diferencias según de quien se trate, observamos que en 2013 un 83\% de los españoles pensaba que la ley no se aplica de forma igual para todos. Ese porcentaje es de un 78\% para el año 2016, si bien los datos para los dos años son bastante semejantes, encontrándose sus porcentajes dentro del intervalo de confianza. Es fácil observar como para una mayoría importante de españoles la igualdad ante la ley es bastante cuestionable. En ese sentido solamente un 14\% en 2013, y un 17\% en 2016 aceptaba que la ley se aplica de igual modo para todos los ciudadanos españoles. Además, dado lo elevado del porcentaje de ciudadanos que afirma la desigualdad ante la ley, cabe destacar cómo este porcentaje es estructuralmente estable para diferentes variables de control, como son género, edad, ingresos, clase social o educación. Esta transversalidad en la percepción social de que la ley se aplica en forma desigual a los ciudadanos españoles es muy significativa en la medida que habla de una opinión generalizada.

Precisamente, uno de los aspectos más destacados es la transversalidad de las opiniones, de modo que la opinión sobre la aplicación desigual de la ley aparece como un tópico generalizado en la sociedad española. Forma parte de la cultura popular y no son escasos los refranes y sentencias populares que tradicionalmente señalan en ese sentido. Cabe con ello señalar que se detecta una debilidad importante en la estructura institucional del estado español desde el momento que la ley no se percibe aplicada por igual. 
Paloma Alaminos-Fernández y Antonio Alaminos-Fernández

Tabla 2. Opinión acerca de la igualdad de trato en la aplicación de las leyes en España

\begin{tabular}{lrrrc}
\hline & \multicolumn{2}{c}{2013} & \multicolumn{2}{c}{2016} \\
\hline & Frecuencia & Porcentaje & Frecuencia & Porcentaje \\
\hline Se da el mismo trato a todas las personas & 345 & 13,9 & 432 & 17,4 \\
\hline Se hacen diferencias según de quien se trate & 2069 & 83,6 & 1945 & 78,2 \\
\hline N.S. & 56 & 2,3 & 100 & 4 \\
\hline N.C. & 4 &, 2 & 9 & 0,4 \\
\hline Total & 2474 & 100 & 2486 & 100 \\
\hline
\end{tabular}

Fuente: CIS E3000 septiembre de 2013 y E3150 septiembre de 2016

Una vez concluido que la justicia es percibida por la opinión pública como desigual en su trato a los ciudadanos españoles, es el momento de considerar qué grupos o colectivos se consideran por un lado como privilegiados para la acción de la justicia en términos de discriminación positiva y que otros grupos se entienden que son considerados de forma negativa (injusta) en lo que podría considerarse como una discriminación negativa.

En términos generales, se entiende una discriminación positiva cuando se tiene en consideración las diferencias entre los individuos, sus condiciones desiguales y desfavorables, en el momento de ponderar una decisión o acción. En ese sentido, en tanto que positiva, se entiende que favorece a los grupos discriminados para compensar una situación desventajada. Por el contrario, la discriminación negativa refuerza la situación de desigualdad en que se encuentran los individuos o grupos sociales, de forma que profundiza y perjudica aún más a los que la sufren. La base para aplicar la discriminación negativa son los prejuicios, estereotipos negativos o la imagen social negativa que presenten determinadas características individuales o grupos sociales.

\subsection{A quiénes discrimina la justicia}

Para dar respuesta a qué grupos o qué características de los ciudadanos son significativas para que la justicia actúe de forma desigual se han empleado los datos procedentes de dos encuestas realizadas por el Centro de Investigaciones Sociológicas en 2013 y 2016. Así, tras la pregunta que hemos mencionado 
anteriormente sobre la aplicación igual o desigual de la ley, en la encuesta de 2013 se plantean posteriormente, otras dos para los que creen que se hace diferencias ${ }^{2}$ :

¿Qué características cree Ud. que podrían favorecer a una persona a la hora de que se le aplique la ley?

¿Qué características cree Ud. que podrían perjudicar a una persona a la hora de que se le aplique la ley?

Y en la encuesta de 2016, tras usar como filtro la igualdad o desigualdad de la aplicación de la ley, se pregunta únicamente por la discriminación negativa, permitiendo una respuesta múltiple escogiendo todas las características que se considere convenientes por parte del entrevistado ${ }^{3}$.

¿Qué características cree Ud. que podrían perjudicar a una persona a la hora de que se le aplique la ley? Por favor, mencione todas las características que podrían perjudicar, por poco que sea.

Resulta evidente que la pregunta de 2016 obtiene una medición mucho más sensible que el diseño de 2013, especialmente al incluir la aclaración "podrían perjudicar, por poco que sea". Asimismo, en términos de validez es más eficaz, dado que el diseño de 2013 proponía un conflicto dado que para todos los colectivos debía evaluarse tanto la posible discriminación positiva como negativa. El planteamiento mismo inducía a dificultades para comprender su propósito.

En todo caso, el diseño de 2013 permite realizar una exploración (con todas las dificultades consideradas) de las características que según la opinión pública española puede inducir a ser tratado por la justicia de forma favorable.

\subsection{Discriminación positiva}

En este estudio empleamos el término discriminación positiva en un sentido genérico y no sinónimo de la denominada como "acción positiva" o "acción afirmativa". No obstante, apunta, si bien en el plano latente o informal, a la posibilidad de existir, en la aplicación por parte de la justicia (Velasco, 2007) de actuaciones dirigidas a reducir las prácticas discriminatorias en contra de sectores históricamente excluidos, así como a favor de individuos con características causantes de desigualdad social. En ese sentido, nos preguntamos

\footnotetext{
${ }^{2}$ Los criterios de administración de las preguntas son los siguientes. Mostrar tarjeta C. Marcar todas las que la persona entrevistada mencione, teniendo en cuenta que un mismo colectivo no puede estar en las dos columnas.

${ }^{3}$ El criterio de administración de la pregunta es el siguiente. Mostrar tarjeta D. Marcar todas las que la persona entrevistada mencione.
} 
¿puede considerarse que existe una discriminación positiva en la aplicación de la justicia, en el sentido de atenuar las consecuencias de situaciones y características socialmente desfavorables en los individuos o grupos sociales?

Considerando la discriminación positiva se observa como existe una elevada concentración de la opinión que afirma que tener muchos recursos económicos, en definitiva, ser rico, favorece que la ley se aplique de forma desigual y favorable para ellos. En la encuesta de 2013 un 77\% consideraba que el hecho de tener muchos recursos económicos propicia ser tratado de una forma especial y positiva en el momento de aplicarse la ley.

Tabla 3. Características que podrían favorecer a una persona a la hora de que se le aplique la Ley

\begin{tabular}{|c|c|c|}
\hline & \multicolumn{2}{|c|}{2013} \\
\hline & No & Sí \\
\hline Tener muchos recursos económicos & $22,70 \%$ & $77,30 \%$ \\
\hline Ser español/a & $84,70 \%$ & $15,30 \%$ \\
\hline Ser hombre & $88,50 \%$ & $11,50 \%$ \\
\hline Ser extranjero/a & $88,60 \%$ & $11,40 \%$ \\
\hline Ser mujer & $91,20 \%$ & $8,80 \%$ \\
\hline Tener una discapacidad psíquica & $93,10 \%$ & $6,90 \%$ \\
\hline Tener una discapacidad física & $93,40 \%$ & $6,60 \%$ \\
\hline Ser joven & $94,30 \%$ & $5,70 \%$ \\
\hline Ser una persona mayor & $94,80 \%$ & $5,20 \%$ \\
\hline Ser católico/a & $94,90 \%$ & $5,10 \%$ \\
\hline Ser de etnia gitana & $95,30 \%$ & $4,70 \%$ \\
\hline Ser heterosexual & $96,20 \%$ & $3,80 \%$ \\
\hline Ser musulmán/a, judío/a, de otra religión & $97,30 \%$ & $2,70 \%$ \\
\hline Tener pocos recursos económicos & $97,40 \%$ & $2,60 \%$ \\
\hline Estar desempleado/a & $98,00 \%$ & $2,00 \%$ \\
\hline Ser homosexual & $98,60 \%$ & $1,40 \%$ \\
\hline Ser transexual & $99,20 \%$ & $0,80 \%$ \\
\hline
\end{tabular}

Fuente: CIS E3000 septiembre de 2013 
Todos los demás grupos propuestos quedan bastante lejos de dicho porcentaje, con valores mucho más bajos. Así el 15\% afirma que ser español es beneficioso a la hora de ser juzgado, un $11 \%$ que a los hombres se les juzga de forma más favorable que a las mujeres, y otro $11 \%$ que a los extranjeros se les favorece en el momento de aplicar la justicia. Precisamente en el extremo opuesto, en el sentido de ser grupos que se consideran muy poco favorecidos por la justicia se encuentran aquellos que tienen pocos recursos económicos, con un $2 \%$, al igual que estar desempleado. En ese sentido está claro que la riqueza y la pobreza marca las referencias clave para la opinión pública en el sentido de una aplicación igual o desigual de la justicia.

Esta idea aparece nuevamente cuando se considera el caso de la discriminación negativa. Es decir, qué grupos son perjudicados por la justicia a ser tratados de forma desigual, en sentido negativo.

\subsection{Discriminación negativa}

En este caso, el planteamiento es el opuesto, en el sentido de explorar la opinión pública sobre el trato desigual, en términos desfavorables, de aquellos individuos o grupos con determinadas características. La pregunta es ahora ¿Puede considerarse que existe una discriminación negativa en la aplicación de la justicia, en el sentido de agravar su aplicación en los individuos o grupos sociales con características socialmente desfavorables?

Si en la discriminación positiva ocupaba un lugar destacado la tenencia de recursos económicos, cuando se trata de discriminación negativa (injusticia) los recursos económicos ocupan también un lugar importante. Nuevamente, tanto para el año 2013 como para el 2016, destaca como causa de trato injusto por parte de la justicia, en primer lugar, el "tener pocos recursos económicos". En 2013 un 61\% de los españoles consideraba que el tener pocos recursos económicos, en otras palabras, ser pobre, hacía que la justicia les tratase de forma desigual y desfavorable. Esta primera posición se mantiene también para el año 2016, siendo un $69 \%$ los que consideraban que ser pobre perjudicaba ante la justicia. Esta pregunta en el año 2013 se colocó justo detrás de la que preguntaba sobre la discriminación positiva de estos mismos grupos y, en ese sentido, generó porcentajes menores de atribución de discriminación negativa. Aun así, con dichas limitaciones, para el caso de 2013, destacan los grupos formados por ser de "etnia gitana", con un $21 \%$ que considera son tratados de forma desigual y desfavorable, o ser "extranjero" con el $27 \%$. 
Paloma Alaminos-Fernández y Antonio Alaminos-Fernández

Tabla 4. Características que podrían perjudicar a una persona a la hora de que se le aplique la ley

\begin{tabular}{|c|c|c|c|c|}
\hline & \multicolumn{2}{|c|}{2013} & \multicolumn{2}{|c|}{2016} \\
\hline & No & Sí & No & Sí \\
\hline Tener pocos recursos económicos & $38,4 \%$ & $61,6 \%$ & $31,0 \%$ & $69,0 \%$ \\
\hline Ser extranjero/a & $72,3 \%$ & $27,7 \%$ & $42,9 \%$ & $57,1 \%$ \\
\hline Ser de etnia gitana & $68,7 \%$ & $31,3 \%$ & $43,3 \%$ & $56,7 \%$ \\
\hline Ser musulmán/a, judío/a, de otra religión & $83,7 \%$ & $16,3 \%$ & $50,6 \%$ & $49,4 \%$ \\
\hline Ser mujer & $85,2 \%$ & $14,8 \%$ & $51,0 \%$ & $49,0 \%$ \\
\hline Ser homosexual & $83,1 \%$ & $16,9 \%$ & $55,3 \%$ & $44,7 \%$ \\
\hline Ser transexual & $83,8 \%$ & $16,2 \%$ & $56,6 \%$ & $43,4 \%$ \\
\hline Tener una discapacidad psíquica & $86,2 \%$ & $13,8 \%$ & $57,7 \%$ & $42,3 \%$ \\
\hline Tener una discapacidad física & $88,4 \%$ & $11,6 \%$ & $61,7 \%$ & $38,3 \%$ \\
\hline Estar desempleado/a & $88,5 \%$ & $11,5 \%$ & $67,2 \%$ & $32,8 \%$ \\
\hline Ser una persona mayor & $92,5 \%$ & $7,5 \%$ & $71,8 \%$ & $28,2 \%$ \\
\hline Ser joven & $91,9 \%$ & $8,1 \%$ & $77,5 \%$ & $22,5 \%$ \\
\hline Ser hombre & $95,5 \%$ & $4,5 \%$ & $79,6 \%$ & $20,4 \%$ \\
\hline Ser español/a & $94,6 \%$ & $5,4 \%$ & $80,5 \%$ & $19,5 \%$ \\
\hline Ser heterosexual & $98,3 \%$ & $1,7 \%$ & $88,3 \%$ & $11,7 \%$ \\
\hline Ser católico/a & $99,1 \%$ & $0,9 \%$ & $91,2 \%$ & $8,8 \%$ \\
\hline Tener muchos recursos económicos & $97,8 \%$ & $2,2 \%$ & & \\
\hline
\end{tabular}

Fuente: CIS E3000 septiembre de 2013 y E3150 septiembre de 2016

Con unos porcentajes menores, pero también significativos, aparecen entre los grupos desfavorecidos por la justicia, y por lo tanto con un trato desigual a los "homosexuales", "transexuales", "musulmanes y de otras religiones" con un $16 \%$, el ser "mujer" con el 14\%, "tener una discapacidad psíquica" con un 13\%, "tener una discapacidad física" con el 11\% así como estar "desempleado", con un 7\% "ser una persona mayor"; un 8\% opina que se puede ser discriminado por la justicia por el hecho de ser "joven" y, ya con porcentajes muy poco significativos el "ser hombre", "ser español”, "ser heterosexual”, "ser católico" o "tener muchos recursos económicos". Esta configuración que hemos considerado el último lugar, en la que diversos grupos se identifican como poco discriminados, se repite en 2016. 
En lo que se refiere a la posición con respecto a ser discriminados por la justicia, en el año 2016 se define nuevamente un conjunto de características que podrían considerarse como identificadoras del ciudadano "normalizado". Así, la menor discriminación de la justicia se produciría para los siguientes atributos: ser joven, hombre, español, heterosexual y católico, así como por tener recursos económicos. Este vendría a ser el perfil del grupo que se considera menos discriminado al aplicar la ley los jueces.

Continuando con los comentarios anteriores, como hemos dicho para el año 2016, nuevamente un $69 \%$ considera que aquellos que son pobres, es decir con pocos recursos económicos, tienden a ser tratados de forma injusta y desigual por la justicia, seguidos con un 57\% los extranjeros, el 56\% los ciudadanos de etnia gitana, con un 49\% las mujeres o tener una religión diferente (musulmán, judío u otra religión), con el 44\% los homosexuales, el 43\% los transexuales, seguidos por los discapacitados psíquicos, $42 \%$; y con un 38\% los discapacitados físicos. Abundando en la falta de recursos económicos como fuente de discriminación de la justicia, un $32 \%$ considera que estar desempleado hace que se sea tratado de forma desigual por la justicia.

Con carácter general, y en términos de resumen, cabe concluir los siguientes aspectos. En primer lugar, una amplia mayoría de españoles considera que la justicia se aplica de forma desigual a los ciudadanos españoles atendiendo a sus características o circunstancias. En el sentido de un trato favorecedor se identifica claramente a los ricos, es decir aquellas personas con mayores recursos económicos. Este grupo aparece tipificado tanto en positivo, en el sentido de ser discriminado positivamente al ser favorecido por la justicia, como a la inversa, por ser considerado el grupo que en menor grado es tratado de forma injusta.

Entre los más discriminados se encuentra la misma causa, según la opinión pública. La pobreza o falta de recursos es la que causa que determinados colectivos reciban un trato especialmente injusto y desigual por parte de la justicia. En un porcentaje elevado, y de forma consistente para los dos años considerados, la pobreza o el desempleo, identifican aquellos colectivos que son más susceptibles, según la opinión pública, de ser tratados de forma desigual por parte de la justicia española.

Resulta evidente que el principal factor de desigualdad ante la ley es el dinero. Los ricos no son iguales ante la ley (les favorece) del mismo modo que perjudica a los que no lo son (los pobres). La pobreza, la falta de recursos económicos en general, se identifica además como causa de discriminación en todos los ámbitos, más allá de la aplicación de la justicia. Según la opinión pública española, la ley es desigual favoreciendo a los ricos y perjudicando a los pobres. 
En términos de igualdad ante la justicia existen unas concepciones sociales bastante bien definidas. Un aspecto complementario es el estudio de las afinidades entre las características que se consideran discriminadas, en particular en lo referido al a discriminación negativa. Este análisis es viable desde un punto de vista agregado sobre la base de la valoración conjunta de los grupos desde el punto de vista de la opinión pública.

\section{AGRUPACIÓN DE FACTORES DE DESIGUALDAD}

Existen múltiples métodos para analizar las dimensiones subyacentes en la valoración de un grupo de variables. En este caso, se ha recurrido al análisis factorial (Alaminos et al, 2015) como procedimiento multivariante para estudiar las agrupaciones, según la opinión pública, de las características consideradas. Este análisis dimensional se ha efectuado para los dos niveles de estudio considerados: a) la percepción de discriminación desde el ámbito de la sociedad y b) el trato desigual de la justicia según características de los individuos o grupos.

En primer lugar, vamos a considerar la percepción social de la discriminación para, en segundo lugar, estudiar cómo se agrupan las características en la percepción de la desigualdad en la aplicación de la justicia.

\subsection{Discriminación social}

En el análisis de las dimensiones de la discriminación en el ámbito social, se ha realizado un análisis factorial de componentes principales, con rotación oblicua, dado que es poco consistente que las dimensiones subyacentes sean independientes entre sí. Es evidente que los factores que organizan la percepción de la discriminación se encuentren relacionados al ser todas características relacionadas.

El análisis de las características, presentadas en la tabla 1 para los datos 2016, extrae dos factores de agrupación principales. Con autovalores de 5,9 para el primer factor y de 1,2 para el segundo, alcanzan un 54,9\% de la varianza explicada.

Uno de los problemas en el caso de analizar variables latentes es la necesidad de identificar un nombre que sea adecuado a las características que agrupa estadísticamente. Por lo general, es fácil identificar cual es la lógica que agrupa dichas características, siendo lo complicado la asignación de un nombre que la describa en su totalidad. Una cuestión que tampoco es de menor importancia se refiere a las características que se ofrecen para evaluación, que en ocasiones solo definen parcialmente alguna dimensión. 
Tabla. 5. 2016. Varianza total explicada

\begin{tabular}{|c|c|c|c|c|c|c|c|}
\hline \multirow[b]{3}{*}{ Componente } & \multicolumn{3}{|c|}{ Autovalores iniciales } & \multicolumn{3}{|c|}{$\begin{array}{l}\text { Sumas de extracción de cargas al } \\
\text { cuadrado }\end{array}$} & \multirow{3}{*}{\begin{tabular}{|l}
$\begin{array}{l}\text { Sumas de } \\
\text { rotación de } \\
\text { cargas al } \\
\text { cuadrado }\end{array}$ \\
Total
\end{tabular}} \\
\hline & & & $\mathrm{e} \%$ & & & & \\
\hline & Total & varianza & acumulado & Total & varianza & $\%$ acumulado & \\
\hline 1 & 5,936 & 45,659 & 45,659 & 5,936 & 45,659 & 45,659 & 5,240 \\
\hline 2 & 1,212 & 9,321 & 54,980 & 1,212 & 9,321 & 54,980 & 4,717 \\
\hline 3 & 834 & 6,413 & 61,393 & & & & \\
\hline 4 & 815 & 6,266 & 67,659 & & & & \\
\hline 5 & 704 & 5,416 & 73,076 & & & & \\
\hline 6 & 645 & 4,962 & 78,037 & & & & \\
\hline 7 & 597 & 4,596 & 82,633 & & & & \\
\hline 8 &, 559 & 4,297 & 86,930 & & & & \\
\hline 9 & 526 & 4,043 & 90,974 & & & & \\
\hline 10 & 467 & 3,592 & 94,566 & & & & \\
\hline 11 & 330 & 2,540 & 97,106 & & & & \\
\hline 12 & 206 & 1,584 & 98,690 & & & & \\
\hline 13 & 170 & 1,310 & 100 & & & & \\
\hline
\end{tabular}

La primera dimensión viene definida por la discriminación que experimentarían los individuos o grupos con determinadas características distintivas como son la "Orientación sexual" (,879), "Origen étnico o racial" (,816), "Nacionalidad" $(, 791)$, “Identidad sexual (ser transexual)" (,784), "Religión o creencias religiosas" $(, 685)$, "Sexo" (,577) o "Tener determinadas ideas políticas" (,410). Evidentemente, como fuente de discriminación la nacionalidad apela a nacionalidades diferentes a la española, la religión a otras religiones diferentes a la católica, y así sucesivamente. En definitiva, puede afirmarse en base a la información anterior que la fuente de discriminación es la "otredad". Vendría definida por los rasgos del que se puede considerar "exogrupo" en comparación con un patrón normalizado culturalmente. Los otros son los excluidos de la "normalidad" cultural.

En el segundo factor aparece "Tener una discapacidad psíquica" (,895), "Tener una discapacidad física" (,870), "Edad" (,690), “Aspecto físico" (,552), "Tener pocos recursos económicos" $(, 551)$ o "Tener una enfermedad crónica o infecciosa (hepatitis, diabetes, VIH/Sida)" (,491). Son características que, dándose dentro del endogrupo, refieren a una debilidad. Son los no "normales" respecto un

\footnotetext{
${ }^{4}$ Cuando los componentes están correlacionados, las sumas de las cargas al cuadrado no se pueden añadir para obtener una varianza total.
} 
patrón de referencia interno al grupo. Un patrón que tiene que ver con la salud, el aspecto físico, la belleza y la fuerza y la capacidad económica de clase media.

En definitiva, el primer factor de discriminación son los rasgos de exogrupo referidos a un patrón cultural, siendo el segundo referido a rasgos del endogrupo y las desigualdades internas.

Tabla. 6 Matriz de patrón ${ }^{a}$

\begin{tabular}{l|l|l}
\hline & \multicolumn{2}{l}{ Componente } \\
\cline { 2 - 3 } & 1 & 2 \\
\hline Orientación sexual & 879 & \\
\hline Origen étnico o racial & 816 & \\
\hline Nacionalidad & 791 & \\
\hline Identidad sexual (ser transexual) & 784 & \\
\hline Religión o creencias religiosas & 685 & \\
\hline Sexo & 577 & 152 \\
\hline Tener determinadas ideas políticas & 410 & 256 \\
\hline Tener una discapacidad psíquica & & 895 \\
\hline Tener una discapacidad física & & 870 \\
\hline Edad & & 690 \\
\hline Aspecto físico & 161 & 552 \\
\hline Tener pocos recursos económicos & 143 & 551 \\
\hline $\begin{array}{l}\text { Tener una enfermedad crónica o infecciosa (hepatitis, diabetes, } \\
\text { VIH/Sida) }\end{array}$ & 306 & 491 \\
\hline
\end{tabular}

Método de extracción: análisis de componentes principales.

Método de rotación: Oblimin con normalización Kaiser.

a. La rotación ha convergido en 6 iteraciones.

El análisis muestra como desde el punto de vista de la opinión pública son dos las fuentes percibidas de discriminación social: la diferenciación entre exogrupo y endogrupo, y dentro del endogrupo las desigualdades internas respecto a un patrón idealizado culturalmente. En ese segundo factor, ser pobre equivale socialmente a una enfermedad, lo que sugiere la necesidad de un mayor análisis en ese sentido.

Una segunda cuestión importante es determinar, también desde la opinión pública española, cuáles puedan ser lo elementos que configuran la discriminación en el ámbito de la aplicación de la ley. 
Tabla. 7 Matriz de estructura

\begin{tabular}{l|l|l}
\hline \multirow{2}{*}{} & \multicolumn{2}{l}{ Componente } \\
\cline { 2 - 3 } & 1 & 2 \\
\hline Orientación sexual & 836 & 455 \\
\hline Identidad sexual (ser transexual) & 812 & 517 \\
\hline Origen étnico o racial & 806 & 474 \\
\hline Nacionalidad & 755 & 415 \\
\hline Religión o creencias religiosas & 691 & 422 \\
\hline Sexo & 668 & 499 \\
\hline Tener determinadas ideas políticas & 563 & 502 \\
\hline Tener una discapacidad psíquica & 496 & 870 \\
\hline Tener una discapacidad física & 495 & 853 \\
\hline Tener una enfermedad crónica o infecciosa (hepatitis, diabetes, & 601 & 675 \\
VIH/Sida) & & \\
\hline Aspecto físico & 492 & 648 \\
\hline Tener pocos recursos económicos & 474 & 637 \\
\hline Edad & 319 & 633 \\
\hline
\end{tabular}

Método de extracción: análisis de componentes principales.

Método de rotación: Oblimin con normalización Kaiser.

\subsection{Discriminación negativa ante la justicia}

En el análisis de las posibles dimensionalidades en la aplicación desigual de la justicia, tal y como es percibida por la opinión pública, se van a emplear los datos de la encuesta de 2016, en particular las características recogidas en la tabla 4. En el análisis se han retenido cinco factores, dado que las diferencias entre el cuarto y el quinto en términos de autovalor no son significativas. Así mismo, permite un análisis algo más detallado de la relación entre las características consideradas y la percepción de aplicación desigual de la justicia. Los autovalores son los siguientes. El primer factor $(4,404)$, el segundo $(1,693)$, el tercero $(1,209)$, el cuarto $(1,075)$ y el quinto $(, 958)$. Todos ellos con una varianza total explicada del $58,3 \%$. La rotación efectuada ha sido nuevamente oblicua, dado que la previsión entre características es de una asociación significativa.

El análisis de las dimensiones tras las características para las que la opinión pública presume un trato desigual reproduce parcialmente, de forma desagregada, las consideradas en el análisis de la discriminación en el ámbito social. No es posible establecer una relación directa entre ambos análisis dimensionales dado que las categorías son descritas o presentadas de forma diferente. Por ejemplo, no es lo mismo preguntar por "orientación sexual" que "ser homosexual". 
Tabla. 8 Varianza total explicada

\begin{tabular}{|c|c|c|c|c|c|c|c|}
\hline \multirow[b]{2}{*}{ Componente } & \multicolumn{3}{|c|}{ Autovalores iniciales } & \multicolumn{3}{|c|}{$\begin{array}{l}\text { Sumas de extracción de cargas al } \\
\text { cuadrado }\end{array}$} & \multirow{2}{*}{ 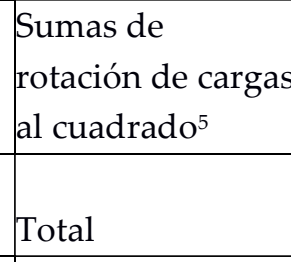 } \\
\hline & eTotal & $\begin{array}{l}\% \text { de } \\
\text { varianza }\end{array}$ & $\%$ acumulado & Total & $\mid \begin{array}{l}\% \text { de } \\
\text { varianza }\end{array}$ & $\%$ acumulado & \\
\hline 1 & 4,404 & 27,525 & 27,525 & 4,404 & 27,525 & 27,525 & 3,470 \\
\hline 2 & 1,693 & 10,584 & 38,109 & 1,693 & 10,584 & 38,109 & 2,387 \\
\hline 3 & 1,209 & 7,558 & 45,667 & 1,209 & 7,558 & 45,667 & 2,879 \\
\hline 4 & 1,075 & 6,720 & 52,387 & 1,075 & 6,720 & 52,387 & 1,231 \\
\hline 5 & 958 & 5,985 & 58,372 & ,958 & 5,985 & 58,372 & 1,540 \\
\hline 6 & 835 & 5,221 & 63,593 & & & & \\
\hline 7 & 824 & 5,149 & 68,741 & & & & \\
\hline 8 & 764 & 4,775 & 73,517 & & & & \\
\hline 9 & 718 & 4,487 & 78,004 & & & & \\
\hline 10 & 640 & 4,003 & 82,007 & & & & \\
\hline 11 & 624 & 3,902 & 85,909 & & & & \\
\hline 12 &, 588 & 3,675 & 89,584 & & & & \\
\hline 13 &, 565 & 3,530 & 93,114 & & & & \\
\hline 14 & 496 & 3,102 & 96,217 & & & & \\
\hline 15 & 335 & 2,092 & 98,308 & & & & \\
\hline 16 & 271 & 1,692 & 100 & & & & \\
\hline
\end{tabular}

Así, el primer factor de discriminación contiene "Tener una discapacidad psíquica" (,790), “Tener una discapacidad física" (,785), "Ser homosexual” (,701) o "Ser transexual" (,674). Corresponde con rasgos individuales y personales, equivalente a un factor de individualidad.

El segundo factor es especialmente interesante, dado que detecta las características de aquellos que no se espera que sufran discriminación negativa por sus características. Representa un factor de "normalidad cultural". Así, este factor está compuesto por "Ser católico/a" (,738), “Ser heterosexual” (,670), "Ser hombre" $(, 657)$ o "Ser español/a" (,645). Todos estos rasgos definen un grupo que se supone excluido de la discriminación negativa puestos a valorar un trato desigual por parte de la justicia.

El tercer factor se refiere a los pertenecientes de forma nítida al exogrupo cultural con características como "Ser extranjero/a" (-,787), "Ser de etnia gitana" (-,755) o

\footnotetext{
${ }^{5}$ Cuando los componentes están correlacionados, las sumas de las cargas al cuadrado no se pueden añadir para obtener una varianza total.
} 
“Ser musulmán/a, judío/a, de otra religión" (-,729). Puede ser denominado como factor de exogrupo cultural.

El cuarto factor habla esencialmente de la pobreza. Así, "Tener pocos recursos económicos" ( ,913) o "Estar desempleado/a" (,526). El factor pobreza es el más mencionado como factor distorsionante en la aplicación de la ley. Recordemos que tanto en términos de discriminación positiva, al aplicar una ley diferente a los más ricos como negativa, al ser más dura con los pobres.

Por último, el quinto factor reúne la edad como elemento de discriminación, tanto por "Ser una persona mayor" (,630) como por "Ser joven" $(, 460)$. Tanto los jóvenes como los mayores son percibidos como características que pueden influir en una aplicación desigual de la justicia. Ambas categorías expresan una posición social débil, unos por no estar integrados aun en la normalidad social (ciclo de vida) y los otros, los mayores, por perder peso y papel social (jubilados) dentro del sistema (trabajo). Factor edad.

Un caso especial es el de la característica "Ser mujer", que con una carga en el primer factor de $(, 319)$ y en el quinto factor de $(, 320)$ se mueve indistintamente entre la discriminación por su sexo (primer factor) y la discriminación por su "debilidad social" (quinto factor).

Tabla. 9 Matriz de patrón ${ }^{a}$

\begin{tabular}{l|l|l|l|l|l} 
& \multicolumn{2}{l}{ Componente } & \multicolumn{2}{l}{} \\
\cline { 2 - 6 } & 1 & 2 & 3 & 4 & 5 \\
\hline Tener una discapacidad psíquica & 790 & & & & 192 \\
\hline Tener una discapacidad física & 785 & & 118 & & 288 \\
\hline Ser homosexual & 701 & &,- 213 & &,- 253 \\
\hline Ser transexual & 674 & &,- 271 & &,- 255 \\
\hline Ser católico/a & & 738 & & & \\
\hline Ser heterosexual & 184 & 670 & & &,- 283 \\
\hline Ser hombre & & 657 & & & \\
\hline Ser español/a &,- 156 & 645 & & & 183 \\
\hline Ser extranjero/a &,- 140 & &,- 787 & & 182 \\
\hline Ser de etnia gitana & & &,- 755 & & \\
\hline Ser musulmán/a, judío/a, de otra religión & & &,- 729 & &,- 126 \\
\hline Tener pocos recursos económicos &,- 105 & & & 913 &,- 156 \\
\hline Estar desempleado/a & 276 & & & 526 & 252 \\
\hline Ser una persona mayor & 222 & & & & 630 \\
\hline Ser joven & & 242 &,- 206 & 141 & 460 \\
\hline Ser mujer & 319 & &,- 255 & & 320 \\
\hline Método dextacciony
\end{tabular}

Método de extracción: análisis de componentes principales.

Método de rotación: Oblimin con normalización Kaiser.

a. La rotación ha convergido en 13 iteraciones. 
Tabla 10. Matriz de estructura

\begin{tabular}{|c|c|c|c|c|c|}
\hline & \multicolumn{5}{|c|}{ Componente } \\
\hline & 1 & 2 & 3 & 4 & 5 \\
\hline Tener una discapacidad psíquica & 793 & 157 &,- 284 & 120 & 316 \\
\hline Tener una discapacidad física & 778 & 173 &,- 231 & & 403 \\
\hline Ser transexual & 766 & 243 &,- 546 & & \\
\hline Ser homosexual & 766 & 239 &,- 500 & &,- 102 \\
\hline Ser mujer & 489 & 246 &,- 431 & & 403 \\
\hline Ser católico/a & 195 & 751 &,- 161 & 115 & 155 \\
\hline Ser heterosexual & 301 & 664 &,- 236 & &,- 135 \\
\hline Ser hombre & 110 & 654 &,- 104 & & 153 \\
\hline Ser español/a & & 630 & & 102 & 266 \\
\hline Ser de etnia gitana & 347 & 110 &,- 763 & & \\
\hline Ser musulmán/a, judío/a, de otra religión & 381 & 166 &,- 760 & & \\
\hline Ser extranjero/a & 210 & 128 &,- 739 & & 223 \\
\hline Tener pocos recursos económicos & & & & 887 &,- 101 \\
\hline Estar desempleado/a & 394 & 225 &,- 213 &, 577 & 359 \\
\hline Ser una persona mayor & 379 & 267 &,- 253 & & 687 \\
\hline Ser joven & 264 & 387 &,- 314 & 215 & 541 \\
\hline
\end{tabular}

Método de extracción: análisis de componentes principales.

Método de rotación: Oblimin con normalización Kaiser.

En definitiva, el análisis dimensional de la actuación desigual de la justicia en función a las características de los individuos muestra una estructura próxima (aunque metodológicamente incomparable) con la de la sociedad en general. En el ámbito de la justicia, destaca la presencia del "factor de normalidad" (factor 3) que identifica a los hombres, heterosexuales, católicos y españoles como los menos susceptibles de ser discriminados. Los otros grupos viene definidos por pertenecer al exogrupo desde el punto de vista cultural (factor 2), por sus características personales que pueden considerarse aún no totalmente normalizadas (factor 1), por la pobreza (factor 4) o por el peso que tienen dentro de la estructura social al ser jóvenes o viejos (factor 5). La mujer aparece clasificada de forma casi indistinta en el factor de rasgos individuales (sexo) o en el de posición social débil (edad).

\section{DEBATE Y CONCLUSIONES}

Existen varias consideraciones que deben hacerse, tanto sobre el diseño de las mediciones como sobre las conclusiones a las que conduce esta investigación. Desde el punto de vista de la formulación de las preguntas destinadas a medir la discriminación positiva y negativa en la encuesta de 2013, es evidente que se produjo un sesgo en la discriminación negativa. No obstante, incluso con dicho 
sesgo se aprecia claramente la estructura de la discriminación en la aplicación de la justicia.

Las preguntas tal y como están formuladas en los dos estudios considerados (2013 y 2016) presentan problemas de comparación debido al planteamiento diferente. A las preguntas en los dos cuestionarios se llega desde el filtro sobre la igualdad ante la ley. Los que afirman que la ley no es igual para todos pasan a la siguiente pregunta, donde se les plantea que características de los juzgados influyen en dicho trato desigual. Estas características o atributos se refieren tanto a la orientación sexual, como a discapacidades, raza, religión, género, nacionalidad o recursos económicos. En definitiva, en las dos preguntas realizadas en los años 2013 y 2016 se explora la relación entre estas categorías y la discriminación en la aplicación de la justicia. La estrategia para plantear la discriminación varía entre 2013 y 2016. En el estudio de 2013 se pregunta primero por aquello que podría contribuir a que la justicia les favoreciera $y$, posteriormente, se pregunta por las características que pueden perjudicar. Además, al considerar que los grupos son excluyentes, el entrevistado no podía identificar una característica como discriminada positivamente por la justicia y posteriormente como negativamente. El orden de las preguntas sin duda influenció en los resultados. Es difícil comprender como se presenta la falta de recursos económicos como una posible ventaja ante la administración de justicia, así como de los demás atributos considerados, que en su mayoría se asocia a la exclusión y la discriminación negativa. Solamente la riqueza es la única característica distintivamente favorecedora de privilegios judiciales.

En el estudio de 2016, comprobado el mal funcionamiento del planteamiento doble favorecer/perjudicar se pregunta exclusivamente por las características que pueden generar un trato desigual por parte de la justicia, en el sentido de perjudicarles. En esta pregunta ya se percibe con nitidez la discriminación a determinados grupos en la aplicación de la justicia.Otro problema metodológico es la falta de comparabilidad entre las categorías. No existe suficiente equivalencia de categorías en los dos tipos de discriminación social y judicial. Como se aprecia en la tabla siguiente, los diferentes fraseos y categorizaciones hacen casi imposible un análisis comparativo entre ellas, dado que plantean realidades muy diferentes.

A pesar de ello, existen indicios que sugieren que la estructura de la discriminación en el ámbito judicial participa, en gran medida, de la estructura de la discriminación en le plano social. Esta afirmación, no obstante, exige de mayor investigación. 
Cuadro 1. Categorías en los dos tipos de discriminación

\begin{tabular}{l|l}
\hline \multicolumn{1}{c|}{ Discriminación sociedad } & \multicolumn{1}{c}{ Discriminación justicia } \\
\hline Orientación sexual & Tener una discapacidad psíquica \\
Origen étnico o racial & Tener una discapacidad física \\
Nacionalidad & Ser homosexual \\
Identidad sexual (ser transexual) & Ser transexual \\
Religión o creencias religiosas & Ser católico/a \\
Sexo & Ser heterosexual \\
Tener determinadas ideas políticas & Ser hombre \\
Tener una discapacidad psíquica & Ser español/a \\
Tener una discapacidad física & Ser extranjero/a \\
Edad & Ser de etnia gitana \\
Aspecto físico & Ser musulmán/a, judío/a, de otra religión \\
Tener pocos recursos económicos & Tener pocos recursos económicos \\
Tener una enfermedad crónica o infecciosa \\
(hepatitis, diabetes, VIH/Sida)
\end{tabular}

Fuente: elaboración propia

En lo que se refiere a las conclusiones, cabe afirmar que la opinión pública entiende que se produce discriminación de los individuos en función a sus características sociales o personales. Entre todas ellas, destaca la pobreza como elemento que facilita y produce discriminación. Una situación igual se reproduce cuando se considera la justicia y su aplicación igual o desigual. La riqueza en el sentido de favorecer como la pobreza en el sentido de perjudicar es el principal rasgo distintivo en la imagen que la sociedad tiene de la administración de la justicia en España. Es una imagen que permanece estable en la cultura española, y que, sin duda, noticias recientes como el fallo favorable a los bancos en sus litigios con los ciudadanos o doctrinas con nombre de banqueros (doctrina Botín) no ayuda a mejorar.

La sociedad en conjunto identifica dos fuentes de discriminación: la "anormalidad" de los identificados como exogrupo frente al endogrupo, y la 
"anormalidad" dentro del endogrupo respecto a un patrón idealizado culturalmente.

En términos de desigualdad ante la justicia, siendo perjudicados, aparecen reagrupadas las categorías anteriores, destacando la identificación del grupo menos perjudicado: hombres, católicos, españoles y heterosexuales. Finalmente, en el establecimiento de dicha desigualdad cabe destacar las dos dimensiones de la discriminación a nivel social: las diferencias entre endogrupo y exogrupo, y las diferencias internas en el endogrupo según su posición o significación dentro de la estructura social. En el ámbito de la justicia, destaca la presencia del "factor de normalidad" (factor 3) que identifica a los hombres, heterosexuales, católicos y españoles como los menos susceptibles de ser discriminados. Los otros grupos viene definidos por pertenecer al exogrupo desde el punto de vista cultural (factor 2), por sus características personales que pueden considerarse aún no totalmente normalizadas (factor 1), por la pobreza (factor 4) o por el peso que tienen dentro de la estructura social al ser jóvenes o viejos (factor 5). La mujer aparece clasificada de forma casi indistinta en el factor de rasgos individuales (sexo) o en el de posición social débil (edad).

En una sociedad que considera que determinadas características discriminan a los individuos, la justicia es percibida dentro de dicho sistema de referencia, como no imparcial. En cierto modo, más allá de la realidad o no de esa aplicación desigual de la ley, la percepción de la sociedad define una situación de deslegitimación de la institución desde el momento que un porcentaje tan elevado como el 78,2\% en 2016 considera que la ley no es igual para todos. La igualdad ante la ley y la aplicación imparcial de la justicia son dos elementos fundamentales en los estados de derecho modernos. En este análisis de la opinión pública española se observa que la percepción mayoritaria de los españoles es de una realidad donde se aplica de forma desigual la justicia. El factor determinante es la capacidad económica de los ciudadanos. Se opina que a los ricos se les favorece y a los pobres se les perjudica.

\section{REFERENCIAS}

Alaminos.Fernández, A. F (2018) Alaminos-Fernández, A. (2018). An ideological conflict: the information about refugees in the European mass media versus the UNHCR communication campaign" Dilemmas". Communication for Development. Malmo University.

Alaminos, A. et al. (2015) Análisis multivariante para las Ciencias Sociales I. Índices de distancia, conglomerados y análisis factorial. Cuenca (Ecuador): Universidad de Cuenca 
Alaminos-Fernández, P. (2015). Crime and future. A new wave punitive Punishment? Flashforward 2015. Global Processes in the Media. Alicante: Universidad de Alicante.

Alaminos-Fernández, A. F. (2015). Nuevas estrategias de medición del desarrollo económico y social. Universitat Jaume I.

Alaminos, A. y Alaminos-Fernández, P. (2014) Estereotipos intraeuropeos sobre el carácter nacional, X JIMEARS. Alicante: OBETS.

Alaminos-Fernández, A. F. (2014). Clases Sociales, Democracia y Estado de Bienestar. Universidad de Alicante.

Alaminos, A. y Alaminos-Fernández, P. (2013) Mapa del etnocentrismo 2011. IX JIMEARS. Alicante: OBETS.

Alaminos, A. y Alaminos-Fernández, P. (2012) Estereotipos sobre los occidentales en países musulmanes. VIII JIMEARS. Alicante: OBETS.

Alaminos-Fernández, A.F. y Alaminos-Fernández, P. (2012). Estereotipos sobre los musulmanes en España, Gran Bretaña, Francia y Alemania. Universidad de Alicante.

Alaminos A. y Castejón J.L. (2006) Elaboración, análisis e interpretación de encuestas, cuestionarios de escalas de opinión. Alcoy: Marfil

Alaminos, A. (1991) Teoría y práctica de la encuesta. Madrid: CEDEAL

Barry, B. (2001): Cultura e igualdad, en Istor, no 7, 38-59.

Baudrillard, J. (2009) La sociedad de consumo. Sus mitos, sus estructuras, Madrid: Ed. Siglo XXI

Boxill, B.R. (1995): Igualdad, discriminación y trato preferente, en Peter Singer (ed.): Compendio de ética, Alianza:Madrid, 457-468.

Dworkin, R. (1984): Los derechos en serio, Barcelona: Ariel.

Dworkin, R. (2003): Virtud soberana. La teoría y la práctica de la igualdad, Barcelona: Paidós.

Foucault M. (2001) Los anormales Madrid: Akal

Francés, F et al. (2014) El proceso de medición de la realidad social: La investigación a través de encuestas. Cuenca (Ecuador): Universidad de Cuenca 
Goffman, E. (1994) Internados. Ensayos sobre la situación social de los enfermos mentales, Buenos Aires: Amorrortu

Goffman, E. (1993) La presentación de la persona en la vida cotidiana", Buenos Aires: Amorrortu

Kymlicka, W. (1995): Filosofía política contemporánea, Barcelona: Ariel.

Kymlicka, W. (1996): Derechos individuales y derechos de grupo en la democracia liberal, en Isegoría, nº14, 5-36.

Rawls, J. (1979): Teoría de la justicia, FCE, México.

Velasco, Juan Carlos: «Discriminación positiva, diversidad cultural y justicia.» Daimon. Revista de Filosofía, no 41, 2007

PALOMA ALAMINOS-FERNÁNDEZ es investigadora del programa Música y Sociedad. Especializada en métodos de investigación aplicada a los impactos sociales de las Nuevas Tecnologías, prospectiva y movimientos sociales. Su última publicación, Los géneros de la música de género en "streaming": un estudio sobre identidad sexual y subcultura musical. Creative Industries Global Conference (CIGC). Máster en Investigación Criminal y Ciencias Forenses (Universidad de Alicante).

ANTONIO ALAMINOS FERNÁNDEZ es graduado en Publicidad y RRPP (Universidad de Alicante), Bachelor en Circumpolar Studies (University of Nordland), Máster Internacional en Paz, Conflicto y Desarrollo (Universitat Jaume I), Master of Arts with a Major in Communication for Development (Malmö Universitet, Sweden) y Titulado en Música, especialidad Violín y Viola. Última publicación: La transformación musical de los no-lugares. OBETS. Revista de Ciencias Sociales 13 (1), 211-228. Especialista en la investigación de la música y sus efectos: Emociones, valores y emociones. Áreas: Música y movimientos sociales, diplomacia de las celebridades, publicidad y música ambiental. Actualmente es investigador del programa "Música y Sociedad" en la Universidad de Alicante. 